\title{
Negando ou Subestimando Problemas Ambientais: Barreiras Psicológicas ao Consumo Responsável
}

\author{
Fabio Iglesias \\ Lucas Soares Caldas \\ Luisa Alcântara Teixeira Rabelo \\ Universidade de Brasilia \\ Brasília, DF, Brasil
}

\begin{abstract}
RESUMO
Embora a busca de soluções para os problemas ambientais seja tipicamente associada à tecnologia e a intervenções de larga escala, mudanças de comportamento no nível individual contribuem diretamente para o consumo sustentável. Este trabalho investigou barreiras psicológicas que as pessoas apresentam para não se comportar pró-ambientalmente nas situações em que poderiam facilmente fazê-lo. Para alcançar este objetivo, uma medida criada com base em 12 dos 29 "dragões da inação" previstos no quadro teórico de Gifford (2011), foi traduzida, adaptada e respondida por 272 participantes. Análises fatoriais sugeriram que as barreiras psicológicas se organizam em dois tipos: (1) negação do problema; (2) prioridades conflitantes. A medida apresentou evidências de validade e fidedignidade. Discutem-se as aplicações desses resultados na promoção da sustentabilidade, que podem envolver ações cotidianas como a economia de energia doméstica, o uso de transporte coletivo e o descarte adequado de lixo.
\end{abstract}

Palavras-chave: Comportamento pró-ambiental; consumo responsável; barreiras psicológicas.

\begin{abstract}
Denying or Underestimating Environmental Problems: Psychological Barriers to Responsible Consumption

Although the search for solutions to environmental problems is typically associated with technology and largescale interventions, behavioral changes at the individual level directly contribute to sustainable consumption. This study investigated psychological barriers that people report for not behaving pro-environmentally in situations where they could easily do it. A measure based on 12 of 29 of the "dragons of inaction" in Gifford's (2011) theoretical framework was translated, adapted and answered by 272 participants. Factor analyses suggested that the psychological barriers have a two-dimensional structure, involving: (1) denial of the problem; (2) conflicting priorities. The measure presented evidences of validity and reliability. Potential applications of these results in the promotion of sustainability are discussed, which can involve everyday actions such as household energy saving, the use of public transportation and proper waste disposal.
\end{abstract}

Keywords: Pro-environmental behavior; responsible consumption; psychological barriers.

\section{RESUMEN}

Negando o Minimizando los Problemas Ambientales: Barreras Psicológicas para el Consumo Responsable

Aunque la búsqueda de soluciones para problemas ambientales se asocia típicamente con la tecnología y las intervenciones a gran escala, los cambios de comportamiento a nivel individual contribuyen directamente con el consumo sostenible. Este estudio investigó las barreras psicológicas que las personas presentan para no comportarse proambientalmente en situaciones en las que podrían hacerlo fácilmente. Una medida basada en 12 de los 29 “dragones de la inacción" en cuadro teórico de Gifford (2011) fue traducida, adaptada y respondida por 272 participantes. Análisis factoriales sugirieron que las barreras psicológicas se organizan en dos dimensiones: (1) negación del problema; (2) prioridades conflictantes. La medida presentó evidencias de validez y confiabilidad. Se discuten las aplicaciones potenciales de estos resultados en la promoción de la sostenibilidad, que pueden incluir el ahorro de energía residencial, el uso del transporte público y la disposición adecuada de la basura.

Palabras-clave: Conducta proambiental; consumo sostenible; barreras psicológicas. 


\section{INTRODUÇÃO}

Apesar do inestimável avanço da psicologia na investigação de atitudes, crenças, valores, preferências e outros construtos relevantes, a efetiva mudança de comportamentos permanece como um dos maiores desafios práticos da área como um todo (Ross, Lepper, \& Ward, 2010). No caso dos comportamentos próambientais, existe uma clara distância que separa, de um lado, o conhecimento que a psicologia ambiental, a psicologia social e a psicologia do consumidor produziram e, de outro, as aplicações que conseguem implementar. É notável, por exemplo, que a American Psychological Association tenha dedicado uma forçatarefa para tratar das interfaces da psicologia com as mudanças climáticas que se observam em nível global, apontando diversos desafios que ainda não têm sido devidamente enfrentados (APA, 2010).

Acordos firmados entre nações com o fim de reduzir os danos ao meio ambiente, ações governamentais e nãogovernamentais, desenvolvimento de novas tecnologias e investimentos de ordem estrutural são aqueles mais destacados nos esforços de sustentabilidade, mas iniciativas focadas no comportamento individual podem ter uma função estratégica e mais imediata (Clayton \& Brook, 2005). Ainda assim, por que é tão desafiador mudar hábitos de consumo e gerar o envolvimento pessoal de consumidores? Esta pesquisa teve o objetivo de investigar barreiras psicológicas que servem como justificativa para que pessoas não se comportem próambientalmente, quando poderiam fazê-lo no contexto do consumo responsável. No entanto, ao invés de focar em grandes mudanças de comportamento, o interesse é nas pequenas (mas relevantes) situações cotidianas que incluem as típicas atividades residenciais, em seu lazer ou trabalho. O cumprimento desse objetivo foi operacionalizado por meio da adaptação de uma medida de barreiras psicológicas ao comportamento pró-ambiental.

\section{BARREIRAS PSICOLÓGICAS AO CONSUMO SUSTENTÁVEL - OS “DRAGÕES DA INAÇÃO” DE GIFFORD}

Com base em fenômenos investigados de maneira relativamente isolada, tanto na psicologia social quanto na psicologia ambiental, Gifford (2008) inicialmente reuniu um conjunto de nove barreiras psicológicas à sustentabilidade quando se consideram os problemas ambientais na lógica de um dilema social. Tomada de maneira mais ampla, no entanto, essa análise deu origem ao que ele chamou de "dragões da inação" (Gifford, 2011), um quadro teórico que descreve 29 barreiras psicológicas para o comportamento pró-ambiental. A referência mitológica a dragões expressa o modo como esses fenômenos dificultam o alcance de metas humanas, portanto uma metáfora para a desafiadora busca por sustentabilidade no consumo dos recursos naturais. Aqui são revisadas 12 das 29 barreiras, tomadas como as potencialmente mais relevantes para a realidade cultural brasileira e por apresentarem a fundamentação teórico-conceitual ou empírica mais robusta: 1 . embotamento ambiental; 2. falta de controle comportamental percebido; 3 . incerteza; 4. negação; 5. reatância psicológica; 6 . conflito de metas; 7. comparação social; 8. falta de identificação com a comunidade; 9 . tokenismo; 10 . hábitos; 11 . risco percebido; e 12. viés otimista.

1. Embotamento ambiental: Aideia de embotamento ambiental (numbness) foi desenvolvida pelo próprio Gifford (1976) e em sua tradução reúne tanto aspectos de dormência como de ignorância. Constitui barreira psicológica porque em situações de sobrecarga de informações tendemos a simplesmente descartar boa parte delas, ainda que signifiquem prejuízo direto (Bawden \& Robinson, 2009). A frequente exposição a uma infinidade de problemas ambientais, como os que caracterizam o cenário de consumo, gera comportamentos de ignorar mensagens relevantes e reações de adaptação que impedem ações mais sustentáveis. Debates sobre o aquecimento global e suas causas antropogênicas mostram claramente como uma parcela da população mundial desconhece a real existência do problema, ou pelo menos o papel humano nessa dinâmica, favorecendo o descomprometimento no plano individual.

2. Falta de controle comportamental percebido e

3. Incerteza: Na teoria do comportamento planejado de Fishbein e Azjen (2010), a primeira é uma das variáveis fundamentais para aumentar o poder preditivo da atitude sobre a ação. Ele se refere à crença sobre fatores que podem facilitar ou dificultar nossa performance. No caso de comportamentos pró-ambientais, a falta de controle comportamental percebido pode constituir barreira psicológica porque frequentemente inferimos que nosso envolvimento pessoal não pode competir com o que ocorre em nível macro. A incerteza se expressa também porque muitas pessoas argumentam que iniciativas de reciclagem doméstica, por exemplo, são ínfimas perto do que ocorre a nível industrial, de modo que não faz sentido investir tempo, energia ou dinheiro nesses comportamentos aparentemente duvidosos.

4. Negação: De modo relacionado ao dragão da incerteza, uma outra barreira psicológica se refere à simples negação do problema ambiental. Ela tem bases 
freudianas, pois remete a mecanismos de defesa do ego investigados não somente na psicanálise como também inspiradores da literatura contemporânea sobre personalidade. Koger e Winter (2010) examinaram como esses mecanismos nos protegem de sensações de desconforto quando somos confrontados com evidências de degradação do meio-ambiente, via racionalização, intelectualização ou deslocamento, entre outros.

5. Reatância: Ainda por lógica semelhante, outra barreira psicológica se expressa naquilo que Brehm e Brehm (1981) chamaram de reatância psicológica: reagimos negativamente a imposições que parecem restringir nossa liberdade de escolha e nossa autonomia (Levav \& Zhu, 2009). Como muitos apelos para a promoção do comportamento pró-ambiental derivam de leis, campanhas públicas ou até mesmo de empresas, as pessoas podem desenvolver considerável resistência a tentativas de persuasão e até se comportar de modo contrário ao esperado.

6. Conflito de metas: Ainda que muitas pessoas reconheçam a importância de posturas mais sustentáveis de consumo, elas podem se justificar pelo fato de que competem com outras ações mais prioritárias em suas rotinas, constituindo a barreira psicológica de conflito de metas. Ela é fundamentada não somente na literatura sobre valores (Schwartz, 2012), mas também por teorias mais recentes que envolvem enquadramento de metas (Steg, Bolderdijk, Keizer, \& Perlaviciute, 2014). De modo geral, os apelos para se melhor gerenciar recursos naturais significam um sacrifício individual, como reduzir o tempo no banho quente, gastar mais dinheiro com produtos orgânicos ou abrir mão do conforto de um veículo particular para transporte. Além disso, metas de conservação podem ser contraditórias, em sua origem, com aquelas mais amplas que envolvem metas de acumulação de bens e prosperidade.

7. Comparação social: Processos de comparação social expressam outra barreira psicológica relevante, pois o engajamento e a manutenção de comportamentos pró-ambientais estão sujeitos à influência direta de outras pessoas. Se percebemos que estamos nos esforçando mais em relação aos outros, a reação é tipicamente de desmotivação. "Por que devo continuar economizando energia se meus vizinhos parecem deixar as luzes de casa acesas sem necessidade?". Os estudos com base na teoria da norma focada de Cialdini (2011) mostram como a saliência de normas descritivas (o que os outros estão fazendo) e de normas injuntivas (o que é o certo a ser feito) podem competir durante tentativas de influência social. Diversos experimentos com comportamentos pró-ambientais envolvem justamente o marketing de normas sociais, para romper crenças equivocadas na comparação social (Goldstein \& Cialdini, 2009).

8. Falta de identificação com a comunidade: Tem base em processos grupais e no conceito de apego ao lugar, desenvolvido na própria psicologia ambiental (Felippe \& Kuhnen, 2012). Ainda que esta não seja sempre um preditor de comportamento pró-ambiental (Gifford, 2011), Lewicka (2011) argumenta que isso deve ser resultado da maior ênfase dos estudos em apenas um dos elementos que constituem a estrutura tripartite do apego ao lugar, conhecendo-se mais sobre variáveis pessoais do que as variáveis do próprio lugar e os processos que as organizam.

9. Tokenismo: também pouco investigado, o termo é amplamente utilizado nas discussões sobre ações afirmativas ao se referir a pequenas concessões que são feitas por pessoas ou instituições para responder a críticas de que não fazem a inclusão de membros de minorias sociais (Blair \& Smith, 2010). Mas no caso do comportamento pró-ambiental ele pode se expressar em pequenas concessões que fazemos, com baixos benefícios à sustentabilidade, como compensação para nossas ações mais danosas ao meio-ambiente ("eu reutilizo o copo plástico quando tomo água, então posso dirigir um carro que consome muito combustível").

10. Hábitos: A literatura sobre hábitos mostra que eles são uma disposição psicológica para repetir comportamentos passados (Neal, Wood, Labrecque, \& Lally, 2011), constituindo outro relevamte dragão identificado por Gifford. Os hábitos têm relação direta com os comportamentos de sustentabilidade, uma vez que as pessoas podem ser simplesmente inertes em relação a uma mudança de comportamento, por hábito, ou porque seria muito custoso, física ou psicologicamente, mudar o que já fazem (Verplanken \& Roy, no prelo). Hábitos têm sido particularmente estudados na literatura sobre saúde, consumo de energia e transporte público (Cristo, no prelo), temas que direta ou indiretamente envolvem problemas ambientais.

11. Risco percebido: Fora o desafio dos hábitos, outra barreira psicológica é que as pessoas podem perceber muitos riscos potenciais numa mudança de comportamento. Consumo mais responsável pode envolver gastos, confiar em novos produtos ou novas empresas, substituir eletrodomésticos, investir tempo e até mesmo correr o risco de estar fazendo algo em vão, recebendo desaprovação social.

12. Viés otimista: Finalmente, viés otimista constitui uma barreira psicológica que encerra fenômenos resumidos no debate sobre aquilo que se nomeia em Inglês como doomers vs boomers (Koger \& Winter, 2010). Por um lado, a perspectiva dos doomers é aquela de simplesmente adotar um 
pessimismo generalizado, acreditando que nada pode ser feito para solucionar ou pelo menos minimizar os problemas ambientais. Recursos naturais, crescimento desordenado da população mundial e aquecimento global são vistos então como inevitáveis consequências do desenvolvimento humano. Por outro lado, muitas pessoas acreditam que mesmo que haja um problema ambiental de ordem maior, o planeta eventualmente se recuperará sozinho e não é necessária qualquer mudança de comportamento humano nessa direção. É a perspectiva dos boomers, tratada como um otimismo ingênuo e que dá bases para um comportamento mais inconsequente. Ambas posturas são radicais e a maior parte da população se encontra em algum ponto de um contínuo entre elas, mas o viés otimista parece ser uma barreira mais lógica ao consumo responsável e até mesmo estudos em neurobiologia têm buscado as explicações cerebrais para sua ocorrência (Shah, 2012).

\section{A MENSURAÇ̃̃O DAS BARREIRAS PSICOLÓGICAS}

Além do desenvolvimento teórico, as primeiras tentativas de se mensurar de forma conjunta essas barreiras foram realizadas no Canadá por Gifford, Iglesias e Casler (2009) e por Iglesias, Gifford e Casler (2009), que desenvolveram a escala Individuals' Obstacles to Proenvironmental Behavior (IOPB). Esses autores elaboraram 43 itens, expressando 13 dos 29 dragões identificados por Gifford, assim como os oito mecanismos de desengajamento moral de Bandura (1999). Verificou-se que apesar da complexidade e diversidade desses itens, em análises fatoriais elas tenderam a se organizar em três grandes dimensões: Ceticismo sobre a relevância dos problemas ambientais; Apelo a outras prioridades que competem com o comportamento pró-ambiental; e Comparações desvantajosas com outras causas que supostamente explicam melhor os problemas ambientais. Embora essa medida tenha apresentado índices psicométricos robustos e evidências de validade convergente e divergente com outras medidas tradicionais na área, é preciso considerar que o contexto cultural canadense da coleta de dados se caracteriza por um alto grau de preocupação ambiental (Kennedy, Beckley, McFarlane, \& Nadeau, 2009). De fato, esse país é reconhecido por iniciativas relativamente avançadas no que diz respeito a transporte público nos centros urbanos, obrigatoriedade da reciclagem de lixo, controle de agrotóxicos, taxação de consumo de água e outras políticas públicas para fomentar o consumo responsável. O Canadá também se destaca por estar atrás apenas da Finlândia e da Suécia no Índice de Sustentabilidade Ambiental (Environmental Sustainability Index - ESI) utilizado pelo Fórum Econômico Mundial.

Como essas barreiras psicológicas são então percebidas e utilizadas no contexto brasileiro? Preocupações pró-ambientais se manifestam de forma consideravelmente distinta em nossa cultura (Schultz, Gouveia, Cameron, Tankha, Schmuck \& Franek, 2005) e são obviamente influenciadas pelo fato de que a abundância de muitos recursos naturais não estimula o consumo responsável e não se desenvolveram ações robustas para promoção da sustentabilidade. Ainda que o valor preditivo de valores já tenha sido investigado (Coelho, Gouveia, \& Milfont, 2006) e outras variáveis relevantes para explicar comportamentos de conservação, pouco se conhece sobre as justificativas que os brasileiros usam para não agir mais pró-ambientalmente. Este trabalho centrou, portanto, na primeira investigação brasileira de barreiras psicológicas (ou dragões da inação) ao comportamento pró-ambiental, com base em uma medida multifacetada e buscando evidências de fidedignidade e validade para seu uso.

\section{MÉTODO}

\section{Participantes}

Responderam ao instrumento 272 participantes $(58,3 \%$ mulheres $)$, com idades que variaram de 17 a 55 anos $(\mathrm{M}=24,0 ; \mathrm{DP}=9,0)$. A amostra envolveu tanto estudantes universitários $(69 \%)$ quanto transeuntes em um centro comercial urbano, abordados durante o período de setembro a novembro de 2009.

\section{Instrumentos}

Foram utilizadas duas escalas na forma de autorrelato, acompanhadas de um questionário sociodemográfico, que incluiu itens sobre sexo, idade e outras variáveis relevantes dos participantes.

\section{Escala de Barreiras Psicológicas}

A escala IOPB foi traduzida e adaptada da original canadense (Iglesias, Gifford, \& Casler, 2009). Atendendo a critérios fundamentais para a adaptação de instrumentos na pesquisa transcultural (Vijver \& Leung, 2011), a tradução da IOPB do Inglês para o Português foi inicialmente realizada pelos autores, seguida da retrotradução desta versão para o Inglês por um colega bilíngue. Os itens da escala retraduzida foram comparados aos itens correspondentes da escala original e não se verificaram modificações substanciais, 
mantendo a validade de face dos itens. Essa versão traduzida foi submetida a um estudo piloto, realizado com 31 participantes, que forneceram evidências de validade semântica.

Mantiveram-se os 43 itens originais, apresentados na forma de justificativas para o participante não se comportar de forma pró-ambiental. Antes da apresentação dos itens da Escala de Barreiras Psicológicas, foi solicitado ao participante que indicasse algum comportamento pró-ambiental para servir como referência para responder aos itens da escala ("Por favor, escreva alguma coisa que você acha que poderia estar fazendo para se comportar de maneira mais adequada em relação ao meio ambiente:"). Em seguida, tendo este comportamento pró-ambiental como referência, os participantes indicaram a sua concordância com cada item numa escala tipo likert de cinco categorias $(1=$ Discordo totalmente a $5=$ Concordo totalmente) .

\section{Escala Revisada do Novo Paradigma Ecológico (rNEP)}

Para testar evidências de validade convergente utilizou-se uma versão traduzida da revised New Environmental Paradigm (Dunlap, Van Liere, Mertig, $\&$ Jones, 2000). A escala é composta originalmente por 15 itens, avaliados em cinco categorias tipo likert $(1=$ Discordo totalmente até $5=$ Concordo totalmente), que apresentam a concordância entre o novo paradigma ecológico e o antigo paradigma, também chamado de social dominante.

\section{Procedimentos}

Os participantes foram abordados individualmente e convidados a participar de uma pesquisa sobre o meio-ambiente. Nem o convite nem as instruções usaram termos como sustentabilidade ou consumo responsável, para minimizar qualquer influência dessas preocupações, uma vez que elas poderiam ter algum efeito de priming. A ordem de aplicação das escalas foi contrabalanceada, para verificar o efeito da ordem de aplicação das escalas. Para investigação dos itens e de potenciais dimensões que os organizassem, os dados foram analisados por meio de procedimentos descritivos, correlações, alpha de Cronbach, análises de variâncias e análises fatoriais.

\section{Considerações Éticas}

Todos os cuidados éticos relevantes foram seguidos no desenvolvimento da pesquisa e não envolveram qualquer tipo de questão sensitiva, dada a natureza do tema e dos procedimentos de autorrelato utilizados.

\section{RESULTADOS}

Inicialmente o banco de dados foi submetido a um procedimento de análise exploratória, para detectar possíveis casos extremos e a normalidade da distribuição das variáveis. Considerando o tamanho da amostra e a relativa robustez de procedimentos de redução de itens quando alguns não apresentam distribuição normal no contexto de análises fatoriais (Hair, Anderson, Tatham, \& Black, 2005), itens inadequados foram excluídos apenas com base em suas cargas fatoriais reduzidas. Análises dos componentes principais foram realizadas para se investigar preliminarmente a dimensionalidade da medida e a fatorabilidade dos itens $(\mathrm{KMO}=0,85$; alfa total $=0,91)$. No entanto, tendo em vista os resultados da versão original da IOPB no Canadá e a existência de fundamentação teórico-conceitual para organização dos itens, optou-se pelo uso de análises dos eixos principais, testando-se soluções uni, bi, tri e tetradimensionais. Dada a esperada correlação entre os fatores, que não têm motivo para apresentar ortogonalidade, essas análises foram conduzidas com rotações oblíquas.

Seis itens apresentaram cargas fatoriais menores que 0,30 e foram excluídos após se avaliar também sua pertinência. Embora uma solução tridimensional tenha apresentado índices psicométricos aceitáveis, uma solução bidimensional se mostrou muito superior, não somente pelo maior valor das cargas e dos alfas, mas também conceitualmente e por gerar melhor equilíbrio no número de itens por fator, explicando $30 \%$ da variância total. O primeiro deles $(\mathrm{alfa}=0,87)$ manteve 18 itens relacionados principalmente ao desconhecimento, incerteza ou negação do problema ambiental. Foi então nomeado como "Negação do Problema Ambiental" e incluiu itens como: "Não ouvi argumento convincente sobre porque deveria mudar"; "Não me falaram porque eu deveria mudar"; e "Não vejo benefício nessa mudança". O segundo fator (alfa = $0,85)$ manteve 19 itens relacionados principalmente a hábitos, metas conflitantes e normas sociais. Foi então nomeado como "Prioridades Diferentes do Comportamento Pró-Ambiental" e incluiu itens como: "Não tenho sido capaz de mudar meus hábitos"; "Não tenho tempo para isso"; "Não gosto de ser pressionado a fazer mudanças como essa"; e "As pessoas ao meu redor não mudaram". A Tabela 1 apresenta a distribuição dos itens, suas cargas fatoriais e os índices de fidedignidade. Os dois fatores se correlacionaram negativamente, $r=-0,44$. Destaca-se também que o fator "Prioridades Diferentes" predominou nas respostas, apresentando médias mais altas $t(269)=17,39$; $p<0,001$. 
TABELA 1

Cargas Fatoriais, Comunalidades, Alfas de Cronbach da Escala de Barreiras Psicológicas ao Consumo Responsável

\begin{tabular}{|c|c|c|c|}
\hline Item & $\begin{array}{l}\text { Fator } 1-\text { Negação } \\
\text { do problema }\end{array}$ & $\begin{array}{c}\text { Fator } 2 \text { - Prioridades } \\
\text { diferentes }\end{array}$ & $h^{2}$ \\
\hline 17 Não ouvi argumento convincente sobre porque deveria mudar. & 0,68 & & 0,45 \\
\hline 33 Não me falaram do porquê eu deveria mudar. & 0,67 & & 0,41 \\
\hline 31 Não há boas evidências mostrando os benefícios dessa mudança. & 0,65 & & 0,41 \\
\hline 28 Não vejo benefício nessa mudança. & 0,62 & & 0,43 \\
\hline 03 Apenas falsos especialistas promovem essa mudança. & 0,60 & & 0,35 \\
\hline 08 Isso não é minha responsabilidade. & 0,59 & & 0,39 \\
\hline 29 Não ouvi falar que deveria fazer isso. & 0,58 & & 0,32 \\
\hline 14 Não estou certo de que meu comportamento seja realmente problema. & 0,54 & & 0,28 \\
\hline 24 Não tenho certeza sobre como isso ajudaria. & 0,49 & & 0,30 \\
\hline 07 Não me identifico com este lugar. & 0,48 & & 0,24 \\
\hline 11 Não é meu trabalho melhorar este lugar. & 0,47 & & 0,32 \\
\hline 15 Não me sinto parte desta comunidade. & 0,47 & & 0,25 \\
\hline 40 Não seria justo já que as outras pessoas não estão mudando. & 0,46 & & 0,30 \\
\hline 04 Fazer isso não resolveria o problema. & 0,45 & & 0,33 \\
\hline 01 Acho que não faria diferença. & 0,43 & & 0,29 \\
\hline 05 Não estou causando mal a ninguém. & 0,40 & & 0,24 \\
\hline 37 Não dá pra querer resolver todos os problemas do meio ambiente. & 0,33 & & 0,16 \\
\hline 38 Estou esperando que meus amigos também mudem. & 0,32 & & 0,09 \\
\hline 19 Não tenho sido capaz de mudar meus hábitos. & & $-0,63$ & 0,38 \\
\hline 06 Eu teria que me esforçar muito. & & $-0,61$ & 0,35 \\
\hline 10 Não tenho tempo pra isso. & & $-0,61$ & 0,44 \\
\hline 02 Para mim é muito difícil. & & $-0,58$ & 0,30 \\
\hline 18 Isso ocuparia meu tempo livre. & & $-0,58$ & 0,40 \\
\hline 09 Preciso de tempo para pensar sobre como fazer isso. & & $-0,57$ & 0,32 \\
\hline 27 Prefiro fazer outras coisas que eu gosto mais. & & $-0,54$ & 0,44 \\
\hline 42 Mudar traria algumas desvantagens para mim. & & $-0,53$ & 0,25 \\
\hline 30 Isso exigiria uma mudança na minha agenda. & & $-0,51$ & 0,27 \\
\hline 32 Estou muito ocupado para pensar nisso. & & $-0,50$ & 0,47 \\
\hline 21 Não gosto de ser pressionado a fazer mudanças como essa. & & $-0,45$ & 0,37 \\
\hline 20 Outras coisas são mais importantes agora. & & $-0,44$ & 0,35 \\
\hline 43 Não sei como fazer isso. & & $-0,39$ & 0,26 \\
\hline 26 Os resultados do meu esforço para mudar não seriam visíveis. & & $-0,38$ & 0,27 \\
\hline 35 As pessoas ao meu redor não mudaram. & & $-0,38$ & 0,20 \\
\hline 41 O que ocorre a nível industrial torna a minha mudança insignificante. & & $-0,36$ & 0,19 \\
\hline 34 Não sou legalmente obrigado a mudar. & & $-0,35$ & 0,13 \\
\hline 23 Ninguém notaria. & & $-0,34$ & 0,24 \\
\hline 36 Já faço a minha parte. & & 0,33 & 0,10 \\
\hline Autovalor & 9,49 & 2,23 & \\
\hline \% Variância Explicada & 22,08 & 5,19 & \\
\hline Número de itens & 18 & 19 & \\
\hline Alpha de Cronbach & 0,87 & 0,86 & \\
\hline
\end{tabular}

Psico, Porto Alegre, PUCRS, v. 45, n. 3, pp. 377-386, jul.-set. 2014 
Os comportamentos de consumo responsável citados pelos participantes como foco para as barreiras psicológicas foram agrupados em três categorias gerais, por sua frequência e semelhança: produção de lixo $(33,1 \%)$; uso de energia $(26,5 \%)$ e transporte $(8,1 \%)$. Outros comportamentos foram citados, mas não constituíram categorias por sua baixa ocorrência e pela alta diversidade nas respostas. Houve também um considerável número de casos omissos para essa pergunta $(21,8 \%)$, ainda que os respondentes tenham respondido plenamente aos 43 itens da escala. Não foram encontradas diferenças significativas para os fatores em função das categorias de comportamento, quando consideradas apenas essas três mais substanciais. Segundo o critério do lambda de Wilks, o segundo fator (Prioridades Diferentes), gerou os maiores escores, $F(1,231)=227,34, p<0,001, \eta_{\mathrm{p}}{ }^{2}=0,50$. Esses resultados não diferiram em função do sexo dos participantes, $F(1,231)=2,97, p=0,09, \eta_{p}{ }^{2}=0,01$. Nenhuma outra variável sociodemográfica se mostrou relevante nessas comparações de média.

A escala Novo Paradigma Ambiental Revisada (rNEP) apresentou uma solução unifatorial com baixas qualidades psicométricas $(\mathrm{KMO}=0,698$, alfa $=0,67$, após cinco itens terem sido excluídos por apresentarem cargas menores que 0,30. Verificou-se uma correlação negativa entre seus escores e os escores totais da Escala de Barreiras Psicológicas, $r=-0,34$, $p<0,01)$. Finalmente, não se verificou efeito da ordem das escalas. Esses resultados se mantiveram tanto na análise dos escores totais da Escala de Barreiras Psicológicas, $t(237)=0,05, p=0,96$, quanto na análise dos escores do primeiro, $t(237)=0,42, p=0,68$, e do segundo fator, $t(237)=0,15, p=0,88$.

\section{DISCUSSÃO}

O objetivo desta pesquisa foi investigar barreiras psicológicas para o consumo responsável no contexto brasileiro quando ele se manifesta via comportamentos pró-ambientais, o que envolveu a adaptação da escala Individuals' Obstacles to Proenvironmental Behavior. Os resultados ofereceram evidências de diferentes tipos de validade para a medida e das barreiras psicológicas, mas especialmente de construto via análise fatoriais, assim como evidências robustas de fidedignidade. Entretanto, as barreiras tiveram aqui uma estrutura bidimensional, que diferiu da original. $\mathrm{O}$ estudo de Gifford, Iglesias e Casler (2009) identificou um terceiro fator que nomearam como Comparações Desvantajosas, cujos itens foram distribuídos na presente pesquisa nos dois fatores encontrados: Negação do Problema e Prioridades Diferentes. O fato do segundo fator ter gerado maiores escores de concordância pode ser visto como promissor, sinalizando menor apelo à negação dos problemas ambientais, seja em bases legítimas ou hipócritas. Mas lidar com argumentos sobre outras prioridades pode ser ainda mais difícil em processos de mudança que envolvam persuasão do consumidor, pois exigem forte mudança de hábito (Verplanken \& Roy, no prelo).

Procedimentos de redução de itens, como no caso de análise dos componentes principais e análises fatoriais, são fundamentais para se reunir evidências de validade mas podem também diminuir a atenção à diversidade dos construtos envolvidos. $\mathrm{O}$ próprio Gifford (2011) discute o dilema de se adotar uma análise mais parcimoniosa ou mais abrangente no estudos dos dragões. Não se verificou aqui, por exemplo, quais barreiras receberam os maiores e os menores escores, pois não foram representadas de forma balanceada nos itens, não foram tomadas de maneira isolada e ainda se conhece pouco sobre eventuais intercorrelações. Aqui os fatores já revelaram expressiva correlação entre si. Embora tenha enorme valor heurístico e seja já baseado em fenômenos muitas vezes clássicos em psicologia social e psicologia ambiental, em conjunto eles ainda foram pouco investigados e demandam coleta de dados em diferentes contextos e com diferentes comportamentos pró-ambientais.

Apesar dessa nova solução dimensional, observa-se clara correspondência teórico-conceitual nas versões, assim como as barreiras que cada fator expressa pareceram manter o mesmo tipo de organização. É importante notar que a rNEP, aplicada junto com a Escala de Barreiras Psicológicas, revelou qualidades psicométricas relativamente baixas. Ela não pôde garantir, portanto, evidências robustas de validade convergente, mas foram semelhantes a boa parte das adaptações analisadas por Hawcroft e Milfont (2010) em sua revisão sobre os usos e (abusos) da medida em diversas culturas. Trata-se provavelmente de uma limitação da escala original, apesar de ser uma das mais populares na área (Dunlap, 2008), sugerindo que outras mais adequadas sejam utilizadas para investigar relações com as barreiras psicológicas.

Como nas instruções da pesquisa os participantes foram solicitados a indicar um comportamento próambiental que poderiam estar realizando, guiando suas respostas às barreiras psicológicas, destacou-se o fato de que a análise das categorias revelou apenas três mais substanciais: lixo, energia e transporte. Quase um terço das respostas se referiu de algum modo a comportamentos de reciclagem ou descarte adequado do lixo, seja em casa, em ambientes de trabalho ou nas vias públicas. Essas não são 
necessariamente as mudanças pró-ambientais mais fáceis de se implementar no nível individual, mas certamente são melhor operacionalizáveis quando se as compara com as demais, podendo gerar um impacto mais imediato, ainda que estético (Stern, 2008). Por outro lado, mudanças para a economia de energia, que representou cerca de um quarto das respostas, são comportamentos menos visíveis e mais difusos, ainda que os medidores de consumo, por exemplo, gerem feedback quantificável. Algumas intervenções em psicologia social conseguiram mostrar como reduzir o consumo de energia, nesses contextos, com base no marketing de normas sociais (Schultz, Nolan, Cialdini, Goldstein, \& Griskevicius, 2007), mas essa estratégia fica mais restrita ao consumo residencial.

Mudanças relacionadas ao transporte representaram menos de $10 \%$ das respostas, entre aquelas categorizadas, o que parece constituir um grande desafio do ponto de vista aplicado. Existe ainda uma resistência ou simplesmente ignorância dos consumidores sobre o fato de que o transporte individualizado, na forma principalmente de automóveis, constitui ameaças ao meio-ambiente (Gärling \& Steg, 2007). No Brasil esse resultado ganha mais sentido ao se considerarem as políticas de estímulo à compra de automóveis, de acesso facilitado ao crédito e de priorização do transporte rodoviário individual, em detrimento do transporte coletivo ou alternativo. Finalmente, chamou atenção o elevado número de casos omissos nas respostas sobre qual comportamento o participante poderia estar implementando. Ele revela que muitas pessoas simplesmente não sabem o que fazer, espelhando talvez as barreiras psicológicas do embotamento ambiental ou da negação (Gifford, 2011), mas pode ser também um problema de medida, pelo fato de ter sido apresentada em formato aberto. Barros e Pinheiro (2013) mostraram recentemente que adolescentes brasileiros não têm um conhecimento minimamente adequado sobre os fenômenos que envolvem as grandes mudanças climáticas e, ainda mais preocupantemente, seus efeitos locais. Isso tem evidentes implicações para os comportamentos de consumo responsável na próxima geração de consumidores adultos.

Estudos sobre barreiras psicológicas como as aqui investigadas são estratégicos para o desenvolvimento de ações relacionadas ao consumo responsável em diversos níveis de intervenção, em função de como consumidores percebem suas prioridades ou simplesmente negam o problema. No entanto, parece mais adequado, num plano imediato, que se focalize em coisas simples que se podem fazer para melhorar seus comportamentos em direção à sustentabilidade, ao invés de intervenções que dependam de fatores estruturais ou tecnológicos. Gardner e Stern (2008) descreveram, por exemplo, uma lista curta de ações residenciais que constituem significativa diferença no uso de recursos naturais. A mera informação não é suficiente para mudar hábitos, mas se contextualizada para nossa realidade cultural, uma lista assim pode orientar facilmente os brasileiros a como consumir melhor, facilitando a superação de alguns dos dragões da inação.

Osbaldiston e Schott (2012) mostraram em sua meta-análise de 253 intervenções para mudança de comportamento que elas tipicamente funcionam melhor quando combinam várias estratégias ao mesmo tempo e que ainda é difícil isolar seus efeitos para que possam ser generalizados. Promover o consumo responsável, em suas relações diretas ou indiretas com o meioambiente, depende de muitas variáveis que envolvem a intenção de se comportar pró-ambientalmente, normas sociais e normas morais, consciência do problema e comprometimento, entre outros (Bamberg \& Möser, 2007; Lokhorst, Werner, Staats, van Dijk, \& Gale, 2013). Mas alguns modelos explicativos já têm inserido como fatores relevantes as possíveis barreiras a influências positivas no comportamento pró-ambiental (Kollmuss \& Agyeman, 2002). Considerando que a mudança parece mais fácil nos contextos em que os dragões na inação de Gifford são mais fracos, abrese uma importante agenda de pesquisa na interface da psicologia ambiental, da psicologia social e da psicologia do consumidor para a promoção do consumo responsável no plano individual.

\section{AGRADECIMENTOS}

A pesquisa recebeu apoio da FAPDF e do CNPq na forma de equipamentos e da CAPES na forma de bolsa de mestrado para o segundo autor. Os autores agradecem a Amalia Pérez-Nebra, Cecília Weiller Bastos e Raissa Damasceno pela leitura crítica do manuscrito.

\section{REFERÊNCIAS}

American Psychological Association (2010). Psychology and global climate change: Addressing a multifaceted phenomenon and set of challenges. Report of the American Psychological Association Task Force on the Interface Between Psychology and Global Climate Change. Obtido em http://www.apa.org/ science/about/publications/climate-change.aspx

Bamberg, S. \& Möser, G. (2007). Twenty years after Hines, Hungerford, and Tomera: A new meta-analysis of psychosocial determinants of pro-environmental behaviour. Journal of environmental psychology, 27(1), 14-25. doi:10.1016/j. jenvp.2006.12.002

Bandura, A. (1999). Moral disengagement in the perpetration of inhumanities. Personality and Social Psychology Review, 3(3), 193-209. doi: 10.1207/s15327957pspr0303_3 
Barros, H. C. L. \& Pinheiro, J. Q. (2013). Dimensões psicológicas do aquecimento global conforme a visão de adolescentes brasileiros. Estudos de Psicologia, 18(2), 173-182.

Bawden, D. \& Robinson, L. (2009). The dark side of information: Overload, anxiety and other paradoxes and pathologies. Journal of Information Science, 35, 180-191. doi: 10.1177/ 0165551508095781

Blair, I. \& Smith, J. (2010). Tokenism. In J. Levine, \& M. Hogg (Eds.), Encyclopedia of group processes \& intergroup relations (pp. 925-928). Thousand Oaks, CA: Sage. doi: http://dx.doi. org/10.4135/9781412972017.n287

Brehm, J. W. \& Brehm, S. S. (1981). Psychological reactance: A theory of freedom and control. San Diego, CA: Academic Press.

Cialdini, R. B. (2011). The focus theory of normative conduct. In P. A. M. Van Lange, A. W. Kruglanski, \& E. T. Higgins (Eds.), Handbook of theories of social psychology (Vol. 2; pp. 295-312). Thousand Oaks, CA: Sage. doi: http://dx.doi. org/10.4135/9781446249222

Clayton, S. \& Brook, A. (2005). Can psychology help save the world? A model for conservation psychology. Analyses of Social Issues and Public Policy, 5(1), 87-102. doi: 10.1111/j.15302415.2005.00057.x

Cristo, F. (no prelo). Hábito e comportamento de viagem In P. W. G. Taco, I.. L. Neto, L. Santos, \& M. Takano (Orgs.). Comportamento em transportes: Uma abordagem multidisciplinar. São Paulo: Casa do Psicólogo.

Coelho, J. A. P. D. M., Gouveia, V. V., \& Milfont, T. L. (2006). Valores humanos como explicadores de atitudes ambientais e intenção de comportamento pró-ambiental. Psicologia em Estudo, 11(1), 199-207.

Dunlap, R. E. (2008). The New Environmental Paradigm Scale: From marginality to worldwide use. Journal of Environmental Education, 40(1), 3-18. doi: 10.3200/JOEE.40.1.3-18

Dunlap, R. E., Van Liere, K. D., Mertig, A. G., \& Jones, R. E. (2000). Measuring endorsement of the new ecological paradigm: A revised NEP scale. Journal of Social Issues, 56(3), 425-442.

Felippe, M. L. \& Kuhnen, A. (2012). O apego ao lugar no contexto dos estudos pessoa-ambiente: Práticas de pesquisa. Estudos de Psicologia (Campinas), 29(4), 609-617. doi: http://dx.doi. org/10.1590/S0103-166X2012000400015

Fishbein, M. \& Ajzen, I. (2010). Predicting and changing behavior: The reasoned action approach. New York: Psychology Press.

Fransson, N., \& Gärling, T. (1999). Environmental concern: Conceptual definitions, measurement methods, and research findings. Journal of Environmental Psychology, 19, 369-382. doi: http://dx.doi.org/10.1006/jevp.1999.0141

Gardner, G. T. \& Stern, P. C. (2008). The short list: The most effective actions U.S. households can take to curb climate change. Environment, 50, 13-24. doi: 10.3200/ENVT.50.5.12-25

Gärling, T. \& Steg, L. (Orgs.). (2007). Threats from car traffic to the quality of urban life: Problems, causes and solutions. Oxford: Elsevier.

Gifford, R. (1976). Environmental numbness in the classroom. Journal of Experimental Education, 44(3), 4-7.

Gifford, R. (2008). Psychology's essential role in climate change. Canadian Psychology, 49, 273-280. doi:10.1037/a0013234

Gifford, R. (2011). The dragons of inaction: Psychological barriers that limit climate change mitigation and adaptation. American Psychologist, 66, 290-302. doi: 10.1037/a0023566

Gifford, R., Iglesias, F., \& Casler, J. (2009). Development of a measure of individuals obstacles to pro-environmental behaviour $(I O P B)$. Trabalho apresentado no 11th European Congress of Psychology, Oslo, Noruega.
Goldstein, N. J., \& Cialdini, R. B. (2009). Normative influences on consumption and conservation behaviors. In M. Wänke, Social psychology of consumer behavior (pp. 273-296). New York: Psychology Press.

Hair, J. F., Anderson, R. E., Tatham, R., \& Black, W.C., (2005). Análise multivariada de dados. Porto Alegre: Bookman.

Iglesias, F., Gifford, R., \& Casler, J. (2009). Environmentally (ir) responsible behaviour: A measure of psychological justifications. Trabalho apresentado na 2009 Convention da Canadian Psychological Association, Montreal, Canada.

Hawcroft, L. J., \& Milfont, T. L. (2010). The use (and abuse) of the new environmental paradigm scale over the last 30 years: A meta-analysis. Journal of Environmental Psychology, 30, 143-158. doi: http://dx.doi.org/10.1016/j.jenvp.2009. 10.003

Kennedy, E. H., Beckley, T. M, McFarlane, B. L., \& Nadeau, S. (2009). Why we don't "walk the talk": Understanding the environmental values/behaviour gap in Canada. Human Ecology Review, 16(2), 151-160.

Koger, S. M., \& Winter, D. D. (2010). The psychology of environmental problems. New York: Psychology Press.

Kollmus, A., \& Agyeman, J. (2002). Mind the gap: Why do people act environmentally and what are the barriers to proenvironmental behavior? Environmental Education Research, 8(3), 239-260. doi:10.1080/13504620220145401

Levav, J. \& Zhu, R. (2009). Seeking freedom though variety. Journal of Consumer Research, 36, 600-610. doi: 10.1086/ 599556

Lewicka, M. (2011). Place attachment: How far have we come in the last 40 years? Journal of Environmental Psychology, 31 (3), 207-230. doi:10.1016/j.jenvp.2010.10.001

Lokhorst, A. M., Werner, C., Staats, H., van Dijk, E., \& Gale, J. L. (2013). Commitment and behavior change: A metaanalysis and critical review of commitment-making strategies in environmental research. Environment \& Behavior, 45, 3-34. Doi 10.1177/0013916511411477

Neal, D., Wood, W., Labrecque, J., \& Lally, P. (2011). How do habits guide behavior? perceived and actual triggers of habits in daily life. Journal of Experimental Social Psychology, 48, 492-498. http://dx.doi.org/10.1016/j.jesp.2011.10.01

Osbaldiston, R. \& Schott, J. P. (2012). Environmental sustainability and behavioral science: Meta-analysis of proenvironmental behavior experiments. Environment \& Behavior, 44, 257-299. doi: 10.1177/0013916511402673

Paulhus, D. L. (1991). Measurement and control of response bias. In J. P. Robinson, P. R. Shaver \& L. S. Wrightsman (Eds.), Measures of personality and social psychological attitudes (pp. 17-59). San Diego, CA: Academic Press.

Pelletier, L. G., Tuson, K., Green-Demers, I., Noels, K., \& Beaton, A. (1998). Why are you doing things for the environment? The Motivation Toward the Environment Scale (MTES). Journal of Applied Social Psychology, 28, 437-468. doi 10.1111/j.15591816.1998.tb01714.x

Ross, L., Lepper, M., \& Ward, A. (2010). History of social psychology: Insights, challenges and contributions to theory and application. In S. T. Fiske, D. T. Gilbert \& G. Lindzey (Orgs.). Handbook of social psychology ( $5^{\text {th }}$ ed.) (Vol. 1; pp. 3-50). New York: Wiley.

Schultz, P. W., Gouveia, V. V., Cameron, L. D., Tankha, G., Schmuck, P., \& Franek, M. (2005). Values and their relationship to environmental concern and conservation behavior. Journal of Cross-Cultural Psychology, 36, 457-475. doi: $10.1177 / 0022022105275962$ 
Schultz, P. W., Nolan, J. M., Cialdini, R. B., Goldstein, N. J., \& Griskevicius, V. (2007). The constructive, destructive, and reconstructive power of social norms. Psychological Science, 18(5), 429-434. doi: 10.1111/j.1467-9280.2007.01917.x

Schwartz, S. H. (2012). An overview of the Schwartz theory of basic values. Online Readings in Psychology and Culture, 2(1). doi: org/10.9707/2307-0919.1116

Shah, P. (2012). Toward a neurobiology of unrealistic optimism. Frontiers in Psychology, 12(3), 344. doi: 10.3389/ fpsyg.2012.00344.

Steg, L., Bolderdijk, J. W., Keizer, K. E., \& Perlaviciute, G. (2014). An integrated framework for encouraging pro-environmental behaviour: The role of values, situational factors and goals. Journal of Environmental Psychology, 38, 104-115. doi: http:// dx.doi.org/10.1016/j.jenvp.2014.01.002

Stern, P. C. (2008). Environmentally significant behavior in the home. In A. Lewis (Ed.). The Cambridge handbook of psychology and economic behaviour (pp. 363-382). Cambridge: Cambridge University Press.

Verplanken, B. \& Roy, D. (no prelo). Consumer habits and sustainable consumption. In L. Reisch \& J. Thogersen (Eds.).
Handbook of sustainable consumption. Cheltenham, UK: Edward Elgar.

Vijver, F. J. R. van de, \& Leung, K. (2011). Equivalence and bias: A review of concepts, models, and data analytic procedures. In D. Matsumoto, \& F. J. R. van de Vijver (Eds.). Cross-cultural research methods in psychology (pp. 17-45). New York: Cambridge University Press.

\section{Autores:}

Fabio Iglesias - Doutor, Universidade de Brasília.

Lucas Soares Caldas - Mestrando, Universidade de Brasília.

Luisa Alcântara Teixeira Rabelo - Graduada, Universidade de Brasília.

Endereço para correspondência:

Fabio Iglesias

Universidade de Brasília, ICC Sul A1-116

Laboratório de Psicologia Social

CEP 70900-100 Brasília, DF, Brasil

E-mail: iglesias@unb.br

Recebido em: 21.5.2014

Aceito em: 13.8.2014 